\title{
The Integrated Statistical Control Method of Technical Condition of Radio-Electronic Equipment for Prototyping of Robotic Systems
}

\author{
Pavel Budko ${ }^{1}$, Vladimir Goydenko ${ }^{1}$, Aleksei Rozhnov, ${ }^{2, *}$ and Aleksei Vinogradenko ${ }^{1}$ \\ ${ }^{1}$ PJC “Inteltech”, RU-197342, St. Petersburg, Russia \\ ${ }^{2}$ V.A. Trapeznikov Institute of Control Sciences of Russian Academy of Sciences, RU-117997, Moscow, Russia
}

\begin{abstract}
In the article, the approach of integrated statistical control and diagnostics of the radio-electronic equipment, which is based on the analysis, integration of the measuring information arriving from the thermal imager, magnetic field sensors, tension and humidity of air and also statistical control of equipment parameters, is presented. The solution of a control and diagnostics problem is based on monitoring of changes in the average level of a multi-parameter process. Here, Hotelling control charts, integration and processing of the measured information are used to provide the analysis and decision-making on the influence of the operator. The algorithm implements a technique of integrated statistical control of the technical condition of the radio-electronic equipment and is capable to determine the level of its precritical condition. The proposed technique increases the reliability of the identification of technical conditions of radio-electronic equipment and expands the scope of technical means of control and diagnostics.
\end{abstract}

\section{Introduction}

Various Radio-Electronic Equipment (REE) is an important part of the structure of modern technological systems in various branches of industrial electronics and electrical equipment. The value and a relative share of volume of REE in technological systems over the past years continuously increase as well as demands in the creation of effecient systems of control and diagnostics at stages of tests and operation. Nondestructive control is especially important for the uninhabited (unattended) units which are located in remote areas where carrying out control and the REE diagnostic functions by servicemen is impossible. Collecting diagnostic information in similar units has to be carried out in the real-time, without allowing a transition of the precritical Technical Condition (TC) of REE to an accident. The purpose of this work is the development of a new approach of integrated statistical control and diagnostics of REE based on the statistical analysis and an integration of the Measuring Information (MI) received from the thermal imager, sensors of the magnetic field, tension and relative humidity.

Here, we consider a problem of assessment of the TC of objects under control. The developed approach of integrated statistical control of the TC of REE has to meet the following requirements:

1. To carry out an integration of sensor readings so that to define the TC of a unit under control in one parameter "invisible" to one type of sensors, but revealed by sensors of other type;
2. To reveal emergency signals at multidimensional statistical control;

3. To increase the application field of control and diagnostics.

The possibility of realization of the proposed approach relies on use of nondestructive methods of control and diagnostics applied in various branches of industrial electronics and electrical equipment [1-6].

Taking into account the conducted studies of modern approaches and controls for non-residential (sparsely populated) units for control, the task was to develop an approach to the integrated statistical control of the TC of REE, which allows diagnosing the pre-critical and critical state of controlled objects in the real time online regime [7-9].

The stage-by-stage description of process of integrated control of REE is given and the approach to reduction of redundancy of MI in the control system is shown in section two.

The third section describes the reason for low reliability in the existing control systems. The approach of statistical control of the TC of REE based on multidimensional methods of statistical control, which is carrying out correcting of values of the REE parameters during her operation by results of selective control, is established. Separate stages of the approach of integrated statistical control of the TC of REE are presented in the form of the algorithm implementing this approach. In the next sections, the level of the precritical state of the controlled unit is determined, which is identified by the rate at which its controlled parameter exceeds the 
permissible values by obtaining all the relevant measurable data (replenished data set) [10-12].

\section{Integrated control of technical condition of REE}

In the proposed approach the combined essence of control consists in obtaining measuring information on REE based on $j$ hybrid sources (a thermogram, an electromagnetic response, humidity of the air, tension) received, respectively, from the thermal imager, sensors of the tension of magnetic field, the humidity of the air, the voltmeter. Considering that transfer of measuring information on the controlled equipment from sensors on blocks of processing of MI is carried out constantly, during the normal work to the equipment, its volume will be superfluous. For the elimination of redundancy, it is necessary to involve one (two) type of sensors removing information on the most critical for a certain type of equipment to parameters. In those timepoints when controlled parameters go beyond admissible limits, information arriving from sensors will confirm a critical (preemergence) condition of the equipment. In this case, it is important that the data arriving from each sensor supplemented each other gives a fuller picture.

On the basis of the statistical analysis of the measured parameters several samples of REE establish reliability range $D$, i.e. the area of operating states representing an interval of dispersion of values of parameters (proceeding from installation of admissible limits), corresponding to operating state of REE in general. On obviously REE efficient elements on versatile signs create them "a working profile" which is remembered in the form of reference matrixes of the TC. During the current operation (tests) of REE measure instant values of signals from exits of $j$ of sensors, and results of the measured instant values of signals digitize and remember in the form of vectors of the digital sequence $N$ length. From the received vectors of the digital sequence $N$ length, form a matrix of the TC dimension of $\mathrm{N} \times \mathrm{N}$ which is compared to reference matrixes of the TC of “a working profile". Depending on rate of approach to reliability range limits technical condition of the REE is determined.

By results of comparison estimate the TC of the REE element, on the greatest number of coincidence of sectors of the compared reliability ranges to the level of precritical condition and also identify the place of refusal (malfunction) of an element of the equipment. Estimate REE condition at several stages, and at the first stage, use information on places of refusals and conditions of each REE element according to which find a violation of the set functioning mode (normal or critical condition) by comparison with reliability range. At the next stage, the level of the precritical state of the controlled unit is determined, which is identified by the rate at which its controlled parameter exceeds the permissible limits by measuring all available estimation information (data set).

The stages of implementation of assessment of REE, presented in the figure number one in a probabilistic state graph of system display: $P_{1}=1-P_{2}$ is a prior probability of lack of an emergency; $P_{2}$ is a prior probability of emergence of an emergency; $\alpha$ is a false refusal (error of the first sort); $\beta$ is an undetected refusal (error of the second sort); $N$ is a normal work; $A$ is an accident (refusal); $k=1,2, \ldots, K$ are the REE levels. At the same time classes of the TC of REE are designated 1 , $2, \ldots, 5_{1}, 6_{1}$, where $1=P_{2} \bar{\beta}_{0} \bar{\beta}_{P} \ldots \bar{\beta}_{k} ; 2=P_{1} \alpha_{0} \alpha_{P} \ldots \alpha_{k}$; $3=P_{2} \bar{\beta}_{0} \bar{\beta}_{P} \ldots \beta_{k} ; \quad 4=P_{1} \alpha_{0} \alpha_{P} \ldots \bar{\alpha}_{k} ; \quad 5=P_{2} \beta_{0} ; \quad 6=P_{1} \bar{\alpha}_{0} ;$ $5_{1}=P_{2} \bar{\beta}_{0} \beta_{P} ; 6_{1}=P_{1} \alpha_{0} \bar{\alpha}_{P}$. Next, we identify the place of refusal of the REE element.

Classes of the TC of REE, mean the following:

- " 1 ” - the system is blocked, the refusal is found and distinguished;

- " 2 " - the system is efficient, false detection and recognition;

- “ 3 ” - the system is blocked, the refusal is found, but not distinguished;

- " 4 " - the system is efficient, false detection isn't distinguished;

- " 5 " - the system is blocked, the refusal isn't found;

- " 6 " - the system is efficient, recognized as efficient. And, in the figure number one classes of the TC " 1 ”, “2”, “3”, “4” multilevel equipment's of REE belong to the final stage of control, and the classes " 51 ”, “61", etc. are presented at each of $K$ levels.

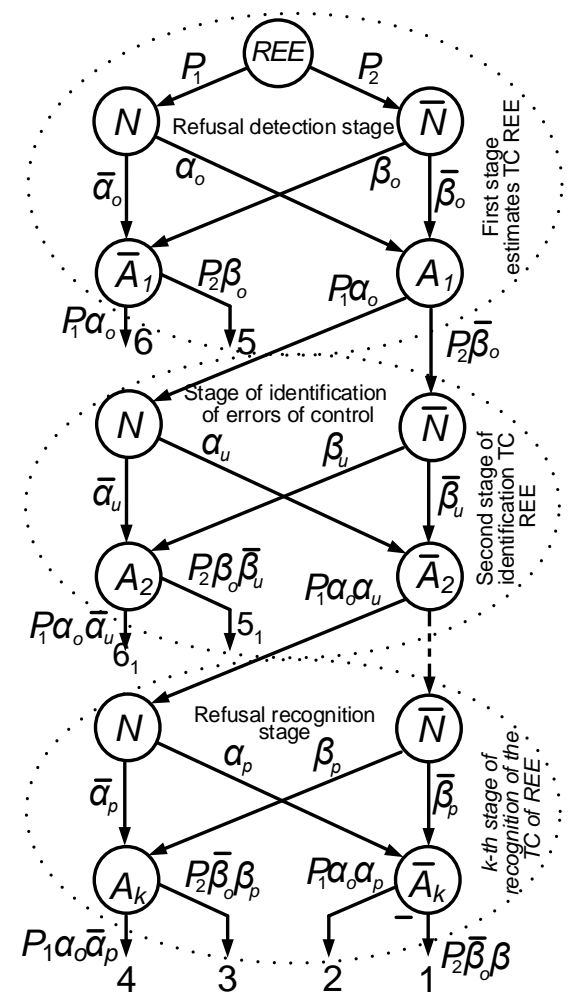

Fig. 1. The probabilistic approach for recognition of the technical condition of REE implementing the stage-by-stage principle of decision-making.

The most preferable is the class " 6 " when the system is efficient and recognized as efficient. The class of technical condition " 1 " goes the following on preference 
- the system is blocked, the refusal is found and distinguished. A variation number of preferences of classes of technical condition of the REE can be written down in a look: "6" > "1" > "4" > "2" > "3" > "5".

\section{Multidimensional statistical control of technical condition of REE}

Control of the REE separate parameters without their interrelations doesn't provide the required size of reliability of control or excessively overestimates operational indicators; at the same time numerous false signals of accident are possible.

Multidimensional Statistical Analysis Methods can be used to control and manage REE for several correlated indicators in checking with Data Envelopment Analysis [7]. Their application means correcting of values of the REE parameters during her operation by results of selective control for maintenance of statistically operated and stable process of work of REE. The developed approach, is based on the analysis and processing of the statistical data arriving in real time from various sensors with REE during the current operation, taking into account tracking of the average level of the correlated indicators for their assessment and decision-making on the operating influence from the operator. So, researches in the interests of substantiating the components are performed in accordance with the Hardwarein-the-Loop (HIL) paradigm. In our case, a HIL simulation includes emulation of sensors and actuators infrastructure of highly automated (intelligent) transport systems [10].

Monitoring of change of the average level of multiple parameter process works of REE are carried out by Hotelling's chart, that is check of a zero hypothesis of equality of a vector of averages to in advance set vector [8]. Its application assumes selection of selections of constant volume at regular intervals and comparison of value of selective statistics with the critical value corresponding to position of control border on the card. Excess of critical value serves as a signal of change of level of control and demands intervention in process of work of REE for his stabilization.

Taking into account controlled $j$ of indicators of multiple parameter process $X_{1}, X_{2}, \ldots, X_{p}$ results of observations of a controlled indicator are analyzed [5-6]: for each instant selection of $t(t=1, \ldots, m)$ the statistics are described

$$
T_{t}^{2}=u\left(\bar{X}_{t}-\mu_{0}\right)^{\mathrm{T}} S^{-1}\left(\bar{X}_{t}-\mu_{0}\right),
$$

where $u$ is a sample size; $\bar{X}_{t}$ is a vector of averages in selection of $t$ (vector assessment $\mu$ ), $\bar{X}_{t}=\left(\bar{X}_{t 1} \ldots \bar{X}_{t p}\right)^{T}, \bar{X}_{t p}$ is an average value in $t$ instant selection on $j$ to parameter $(j=1, \ldots, p) ; \mu_{0}$ is a vector of averages, $\mu_{0}=\left(\mu_{1}, \ldots, \mu_{p}\right)^{T} ; S$ is an assessment of a dispersion matrix $\Sigma$, defining dispersion of indicators of quality and degree of narrowness of their communication,

$$
s_{j k}=\frac{1}{m(n-1)} \sum_{t=1}^{m} \sum_{i=1}^{u}\left(x_{i j t}-\mu_{j}\right)\left(x_{i k t}-\mu_{k}\right) .
$$

At stable process of removal of measuring information the condition $T_{t}^{2}<T_{k p}^{2}$ has to be satisfied, where $T_{k p}^{2}$ is a border of critical area of range of reliability. At assessment a component of a dispersion matrix on a formula (2) position of control limit of the Hotelling's charts decides on use of the current instant selections by a formula

$$
T_{k p}^{2}=\frac{j(m-1)(u-1)}{m u-m-j+1} F_{1-\alpha}(j, m u-m-j+1),
$$

where $F_{1-\alpha}\left(k_{1}, k_{2}\right)$ is a quantile of Fischer distribution with numbers of degrees of freedom $k_{1}$ and $k_{2}$, where $k_{1}$ is in numerator, $k_{2}$ is in a denominator; $\alpha$ is a value of significance value (probability of false alarm).

The main criterion of violation of stability of process is an excess of Hotelling's statistics (1) for control border (3) [4]. However, the use of this criterion does not always provide a sufficiently rapid identification of significant changes in the process under study.

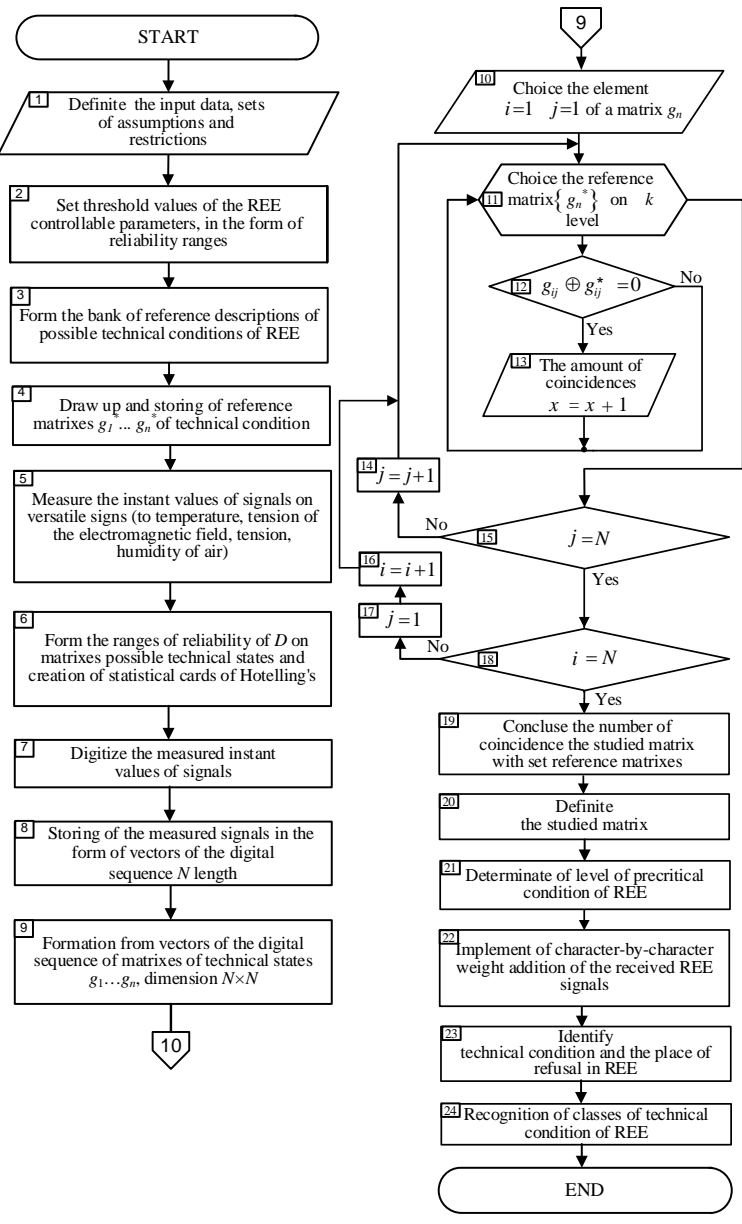

Fig. 2. The algorithm of integrated statistical control of technical condition in overall approach of REE prototyping.

If the deviation of controlled parameters is recorded only in one parameter (for example, humidity of air), and 
others process is in reliability range limits, then the general values of statistics $T_{t}^{2}$ cannot excess over. In this case, for obtaining information on an aberration of the most "critical" parameter in the course of control it is necessary to create not one, but four Hotelling's charts (depending on the number of sensors, controlled parameters), for pairs of parameters.

This gives the opportunity to determinate a deviation of "critical" parameter more clearly relative to others.

The work includes recommendations for system integration of newest vehicles and user services, infoand communication technologies and automation tools with smart infrastructure. The attendant system changes, aimed at providing a set of innovative DEA-services for various modes of vehicle, are presumably designed at achieving sustainable mobility through increasing the efficiency, safety and environmental friendliness of the transport process and improving the infrastructure of highly automated and/or self-driving transport systems.

\section{Conclusion}

The results of the present study show that various control instruments providing an increase in reliability of identification and sensitivity to detection of emergency (preemergency) situations can be used for control of the REE parameters of, especially, autonomous vehicles.

The idea of the complex statistical control of technical conditions of REE presented in the article is based on the integration of several types of sensors and can be used for development of the versatile automated integrated control systems for autonomous vehicles. The corroboration of the developed approach will include prototyping techniques, design approaches, practical applications and implications of robotic systems based on the developed methodologies. In the near future, technologies to support the implementation of such systems and units, as well as related economic, ethical and social considerations, will become a very important component of the intensive growth of multi-layered projects [13-14].

\section{Acknowledgments}

This work was partially supported by the RFBR according to the completed project No.16-29-04326 (hybrid intelligence). The report was partially financially supported by RFBR project No. 19-29-09030 (hybrid intelligence components) and the RSF project No. 17-1101353 (DEA and FDH application). This research was partially based by the Presidium of the Russian Academy of Sciences, Program No. 7 (30) "Theory and Technologies of Multi-level Decentralized Group Control under Confrontation and Cooperation" (investigation of smart infrastructure).

\section{References}

1. D.C. Montgomery, Introduction to statistical quality control (New York: John Wiley and Sons, 2009)
2. S. Ryvkin, A. Rozhnov, I. Lobanov, Proceedings IEEE 20th International Symposium on Electrical Apparatus and Technologies, (SIELA), 8447067 (2018) DOI:10.1109/SIELA.2018.8447067

3. F. Aparisi, J. Jabaloyes, A. Carrion, Comm. in Statistics - Theory and Methods, 28(11), 2671-2686 (1999)

4. Y. K. Chen, K. L. Hsieh, European Journal of Operational Research, 182(3), 1251-1262 (2007)

5. P. A. Budko, G. A. Zhukov, A. M. Vinogradenko, V.K. Goydenko, Sea radio electronics, 58(4), 20-23 (2016)

6. P. A. Budko, A. M. Vinogradenko, A. I. Litvinov, Mechatronics, automation, control, 9(162), 53-58 (2017)

7. A. Lychev, A. Rozhnov, I. Lobanov, Computer Vision in Control Systems-6, Springer, 182, 127139 (2020) doi:10.1007/978-3-030-39177-5_10

8. V. N. Klyachkin, I. N. Karpunina, M. K. Fyodorova, Automation of control processes, 45(3), 58-64 (2016)

9. J. Katzel, Control Engineering, 54(2), 50-54 (2007)

10. A.V. Rozhnov, I.A. Lobanov, Proceedings of 2017 10th International Conference Management of Large-Scale System Development (MLSD), 8109678 (2017) DOI:10.1109/MLSD.2017.8109678

11. S. Ryvkin, A. Rozhnov, I. Lobanov, L. Chernyshov, Proceedings 20th International Symposium on Electrical Apparatus and Technologies (SIELA), 8447069 (2018) DOI:10.1109/SIELA.2018.8447069

12. V. Abrosimov, V. Goncharenko, S. Ryvkin, A. Rozhnov, I. Lobanov, ACEMP 2017, IEEE, 905910 (2017) doi: 10.1109/OPTIM.2017.7975085

13. A. Lychev, A. Rozhnov, Reshetnev-readings, 2, 447-448 (2017) [in Russian]

14. L. C. Jain, M. N. Favorskaya, Computer Vision in Control Systems-6, 182, Springer, 1-6 (2020) doi:10.1007/978-3-030-39177-5 1 